\title{
Study and Application status of Biomagnetic effect in Wastewater
}

\section{Treatment}

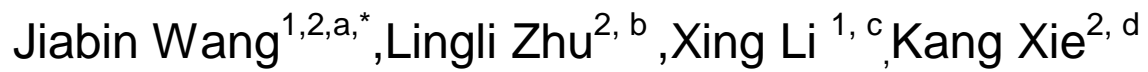 \\ ${ }^{1}$ The College of Architecture and Civil Engineering, Beijing University of Technology, Beijing \\ 100022, China; ${ }^{2}$ School of Architectural and Civil Engineering ,University of Jinan, Jinan 250022 , \\ PR China

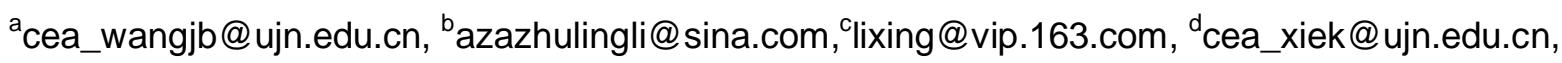 \\ * Corresponding Author \\ e-Mail: azazhulingli@sina.com
}

\section{Keywords:Biomagnetic Effect, Enzyme Activity, Bacterial Activity}

Abstract. Compared with conventional activated sludge system, applied magnetic field or adding magnetic materials in activated sludge system exhibits a better performance of enzyme activity, sludge settling, microbial activity. It also shows an obvious influence on bacterial activity, dissolved oxygen and pollution loadings. Even more, the removal efficiency of organic pollutions, $\mathrm{N}$ and $\mathrm{P}$ in this system shows an obvious increase. The biomagnetic effect is a promising technology in wastewater treatment.

\section{Introduction}

With the exponentially growing population, the water shortage has become the one of the most important problem on the earth. More attention has been paid to the development of high efficient water treatment technology. Many studies indicated that the biomagnetic effect of microorganism is an effective method to strengthen the biological treatment of wastewater. Biomagnetic effect can significantly improve the microbial activity. With applied magnetic field or adding magnetic materials in activated sludge system, the removal efficiency of CODcr is higher than the conventional activated sludge process, the settling of activated sludge exhibits a better performance, and the concentration of activated sludge and the microorganism species increase obviously. Thereby, the biomagnetic effect biomagnetic effect is a promising technology for wastewater treatment.

\section{Biomagnetic effect on enzyme activity}

The main components of the enzyme are $\mathrm{C}, \mathrm{H}, \mathrm{O}, \mathrm{Cl}, \mathrm{S}$ and trace transition metal atoms. Transition metal atoms in many cases show paramagnetic characteristics. Through the impact of the body of the paramagnetic atoms, magnetic field can affect the activity of the enzyme. After adding the magnetic powder in the conventional activated sludge, sludge produces a positive magnetic biological effect. The chemical bonding state and electronic states of the magnetic particle surface are different from interior and the surface atoms are not congruent all of this would lead to the increase of its surface activity to become biologically catalytic agent, which greatly improve the biological activity of microorganisms, oxidation and decomposition of organic pollutants is also a 
corresponding increase.Dai Kelin ${ }^{[1]}$ indicated that adding magnetic powder, magnetic sludge oxygen uptake rate OUR value fluctuated. The increase of magnetic powder dosage from 0 to $1.5 \mathrm{~g}$ dehydrogenase activity showed an increasing trend, and the increase of the magnetic particle dehydrogenase activity increased slightly. Han Qingxing ${ }^{[2]}$ showed the weak magnetic field that under 500 GS can increase the activity of activated sludge microbial enzymes and the refinery wastewater treatment efficiency increased by $11 \%$.

\section{Biomagnetic effect on the performance of sludge}

Adding magnetic substances (e.g., adding magnetic powder) in activated sludge, under the effect of strong magnetic field strength, the settling performance of sludge can improve effectively. Due to the magnetic particle surface with charge, so that the magnetic particles have a strong adsorption to the bacteria in the sludge gradually formed magnetic granular structure compact, reduces the loss of free bacteria and the treatment of the effluent after the light transmission rate increased.

There are research found that in the magnetized activated sludge, sludge proliferation and self -digestion phase offset, thereby inhibiting the proliferation of sludge, there may be zero excess sludge. The mathematical statistics analysis of the influence of the magnetic field on the sludge settling by Li Sheng ${ }^{[3]}$ showed that the magnetic field has an effect on the settling performance of the sludge. The effect of parallel magnetization is larger than that of vertical magnetization. The greater the magnetic field intensity in the parallel magnetization, the greater the impact on the sludge sedimentation performance and the effect of vertical magnetization on the sludge sedimentation performance is not obvious.

\section{Biomagnetic effect on bacterial activity}

Experiments have shown that in magnetic activated sludge can be observed a lot of ciliate, Vorticella and floating worm, a small amount of rotifers, these less in conventional activated sludge, which also increases the sludge of toxic substances in waste water removal capacity. $\mathrm{Li} \mathrm{Jie}^{[4]}$ indicated that the $100 \mathrm{mT}$ field strength showed positive effects on the actinomycetes and fungi, but showed an inhibitory effect on the growth of the bacteria. Wang Qiang ${ }^{[5]}$ showed the magnetic field strength and magnetic way have an impact on the physiological characteristics of anaerobic ammonium oxidizing bacteria. When the external magnetic field strength was $150 \mathrm{mT}$, the removal rate of nitrate and dehydrogenase activity increased $7.2 \%$ and 2.38 times respectively compared with that without magnetic field; Between $30 \sim 90 \mathrm{mT}$, the magnetic field has a promoting effect on the removal of ammonia nitrogen. The better magnetic intensity for ammonia nitrogen removal is $60 \mathrm{mT}$.

Wang ${ }^{[}$Xiangshan ${ }^{[6]}$ study indicated that magnetic field can change the ultrastructure of bacterial cells, magnetic field inside the bacterial cell number of mitochondria increased significantly, and mitochondrial cristae increased, so it can be provide enough spaces for cellular respiration and oxidation reduction.

\section{Biomagnetic effect on dissolved oxygen}

A protein channel is on the microbial cell membrane. External electromagnetic fields through ion channels subjected to the electric field force and the Lorentz force in the role, effect of charged ions of biological membrane penetration of exchange capacity, thereby increasing the mineral water solubility, affecting the organism metabolism, biochemical processes and changes in membrane 
potential, increased water radical; From another perspective, it can increase the dissolved oxygen content of the whole system. Zhang Fan's ${ }^{[7]}$ experimental study shows that under the effect of magnetic effect, the osmotic pressure of water and the concentration of dissolved oxygen in water rise, so that the rate of diffusion of material is accelerated, but also conducive to the absorption and utilization of oxygen by microorganisms.

\section{Biomagnetic effect on the pollution loadings}

Some of the degradation of toxic substances in the microbial generation time is long, the reproduction rate is slow, easy to drain, it is difficult to enrich in the sludge. Therefore, the proportion of these microorganisms in the common activated sludge is small. However, due to the tight structure of the magnetic activated sludge, the magnetic particles provide a suitable habitat for these microorganisms, which is not easy to be lost and gradually enriched. So the load of unit biomass to poison is less than that of common activated sludge. And it can increase the water treatment process of sludge volume load, so can be increased water treatment process of sludge volume load, enable toxic substances increased the removal efficiency, improve water quality, reduce the cost, convenient management.

\section{Biomagnetic effect on wastewater treatment}

Agnieszka $^{[8]}$ in magnetic field enhanced biological wastewater treatment experiment, which a sludge return pipe installation of a permanent magnet fluid in periodic exposed in the 40mT of the permanent magnetic field, another without magnetic, as a non-magnetic system. Magnetic field does not have an obvious effect on the removal of COD, but the transformation of nitrogen has obvious improvement, especially the Kjeldahl nitrogen conversion rate. The study of Raja et al ${ }^{[9]}$. Showed that the biodegradation rate of phenol was greatly improved under the external magnetic field of $22 \mathrm{mT}$. The study of Jung and Sofer ${ }^{[10]}$ indicated that the oxidation rate of phenol was improved in the magnetic field strength of $0.15 \sim 0.35$ T. The research on the treatment of Slaughter Waste Water by magnetic bacteria biological method such as Zhang Milin ${ }^{[1]}$, shows that the treatment effect of waste water produced by slaughter is improved by using nano magnetic part. Wang Chaoyu ${ }^{[12]}$ used ultrafine magnetic $\mathrm{Fe} 3 \mathrm{O} 4$ particles and activated carbon adsorption of activated sludge for the preparation of immobilized microspheres for sewage treatment, it is feasible and effective for the treatment of domestic sewage. Sun Shuiyu ${ }^{[13]}$ used magnetic particle strengthening activated sludge treatment of restaurant wastewater studies have shown that, using a magnetic particle strengthen the sludge treatment of restaurant wastewater, the precipitation speed upgrade, oil removal rate, the CODcr removal and the light transmission of the supernatant rate is better than the conventional activated sludge process. Yavuz ${ }^{[14]}$ found the water treatment with a magnetic field, which efficiency than no magnetic field increase by $44 \%$.

In 2008, Liu Sitong et al. ${ }^{[15]}$ showed that the added magnetic field could increase the activity of anaerobic ammonium oxidizing bacteria in a certain range by a lot of experiments. The maximum activity of anaerobic ammonium oxidizing bacteria under the magnetic field intensity of $75 \mathrm{mT}$ can be increased by about $50 \%$. Liu et al. studied the long term effect of magnetic field on anaerobic ammonia oxidation reactor. Found that the maximum nitrogen removal rate of the reactor can be increased by $30 \%$ under the magnetic field strength of $60 \mathrm{mT}$, and the start-up period of the anaerobic ammonium oxidation can save 1/4 time. 


\section{Conclusions}

The biomagnetic effect has an obvious effect on the process of activated sludge wastewater treatment,and biomagnetic effect also has a lot of research and development space.For example,to investigate whether the biomagnetic effect Play an active role in enriching the anaerobic ammonium oxidizing bacteria. So as biomagnetic effect can promote the application of anaerobic ammonium oxidation process in engineering.

\section{Acknowledgements}

This study was supported by Shangdong Provincial Natural Science Foundation, China (ZR2013EEQ007), and was partly supported by National Natural Science Foundation of China (51278225), Science and technology development projects of Shandong province (2013GSF11704), Shangdong Provincial Natural Science Foundation, China (ZR2015EM021).

\section{References}

[1] Kelin Dai,et al: Resource Saving and Environmental Protection, Vol. 2 (2010), p.41.In Chinese.

[2] Qingxing Han, et al: Journal of Fushun Petroleum Institute, Vol. 22 (2002), p.8.In Chinese.

[3] Sheng Li, et al:Journal of Suzhou University of Science and Technology: Engineering and Technology Edition, Vol. 19 (2006), p.30.In Chinese.

[4] Jie Li, et al: Chinese Journal of Soil Science, Vol. 38 (2007), p.957.In Chinese.

[5] Qiang Wang, et al: China Water \& Wastewater Vol. 26 (2010), p.34.In Chinese.

[6]Xiangshan Wang, et al: Environmental Science \& Technology.Vol. 32(2009), p.112.In Chinese.

[7] Fan Zhang, et al:Modem Chemical Industry. Vol. 23(2003), p.47.In Chinese.

[8] T Agnieszka, Lidia W:Desalination,Vol. 222(2008), p.368.

[9] Raja Rao T B M L, R L Sonolikar, S Pentu Saheb: Chemical Engineering Science, Vol. 52(1997), p.4155.

[10] J.T Jung ,S Sofer . J Chemisty Technology Biotechnology, Vol. 70(1997), p.299.

[11]Milin Zhang, et al: Journal of Harbin Engineering University. Vol. 28 (2007), p.711.In Chinese.

[12]Chaoyu Wang : Master Thesis of Gui Zhou University, 2006.In Chinese.

[13]Shuiyu Sun,et al : Environmental Pollution and Control, Vol. 25 (2003), p.172.In Chinese.

[14] H Yavuz, S.S Celebi: Enzyme and Microbial Technology, Vol. 26 (2000), p.22.

[15]Sitong Liu, et al: Joumal of Biotechnology, Vol. 138 (2008), p.96.In Chinese. 